\title{
Increasing the sensitivity of LXe TPCs to dark matter by doping with helium or neon
}

\author{
W. H. Lippincott* \\ Fermi National Accelerator Laboratory \\ E-mail: hugh@ fnal.gov

\section{T. R. Alexander} \\ Pacific Northwest National Laboratory \\ E-mail: thomas.alexander@pnnl.gov
}

\author{
A. Hime \\ Pacific Northwest National Laboratory \\ E-mail: andrew.hime@pnnl.gov
}

\begin{abstract}
Next generation liquid xenon TPCs are poised to increase our sensitivity to dark matter by two orders of magnitude over a wide range of possible dark matter candidates. This proceedings describes an idea to expand the reach and flexibility of such detectors even further, by adding helium and neon to the xenon to enable searches for very light dark matter and combining high and low $\mathrm{Z}$ targets in the same detector. Adding helium or neon to LXe-TPCs has many advantages. First, the helium or neon target benefits from the excellent self-shielding provided by a large liquid xenon detector. Second, the same instrumentation, PMTs, and data acquisition can be used. Third, light nuclei are more robust to the systematic uncertainties that affect light WIMP searches. Fourth, helium and neon recoils will likely produce larger signals in liquid xenon than xenon recoils, achieving lower energy thresholds, and further increasing the sensitivity to light WIMPs. Lastly, by adding He/Ne in sequence after a Xe-only run, the source of any observed signal can be isolated.
\end{abstract}

38th International Conference on High Energy Physics

3-10 August 2016

Chicago, USA

${ }^{*}$ Speaker. 


\section{Introduction}

The absence of a discovery of new physics in direct dark matter searches and at the LHC has led physicists to imagine a wider range of scenarios to explain the dark matter puzzle, and many of these scenarios predict dark matter particles with masses below $10 \mathrm{GeV}$. Simple extensions to minimal SUSY models can produce WIMP candidates down to $1 \mathrm{GeV}$ that evade all constraints from the LHC [1], and newer ideas have also emerged, as in Asymmetric Dark Matter (ADM) [2]. In ADM models, the fact that the densities of baryonic matter and dark matter are similar to within a factor 5 takes on new significance, and the matter-antimatter asymmetry observed in our universe is produced via interactions with the dark sector. The mass scale for ADM candidates is $\sim 5 \mathrm{GeV}$. For kinematic reasons, low mass dark matter particles do not transfer energy to nuclei as efficiently as heavier WIMPs, particularly for a heavy nucleus like xenon. Therefore, new techniques are required to address these light dark matter models, ideally combining low energy thresholds with a low $\mathrm{Z}$ target.

Recent effective field theory approaches have re-emphasized that there are many potential couplings to dark matter that go beyond the standard spin independent (SI) and spin dependent (SD) cases [3]. These couplings can have very different responses to different nuclei, and searches with multiple targets (even with null results) will be necessary to tease out the true nature of the dark matter interaction [4].

The XENON1T and LZ experiments aim to improve on existing sensitivities by two orders of magnitude for WIMPs above $10 \mathrm{GeV}$. However, as proposed, these are single target experiments, searching for dark matter interactions with xenon nuclei only and limited in sensitivity to low WIMP masses. SuperCDMS-SNOLAB (among others) will focus on the low WIMP mass region with germanium and silicon targets. The primary sensitivity goals of these two classes of experiment do not have much overlap, and they are of necessity limited in the number of nuclear targets used. This proposal outlines a program that will enable LZ or any LXe-TPC to search for dark matter down to $1 \mathrm{GeV}$ and below, providing a strong complement to SuperCDMS and other dedicated light WIMP searches, while also adding the flexibility of running multiple targets in the same detector, for a reasonable cost. By dissolving small concentrations of helium and neon into the LZ detector, we can add a second or third target nucleus that is more kinematically favorable for scattering with low mass WIMPs.

\section{Low mass WIMPs and low energy nuclear recoils}

To understand the sensitivity of a detector for light WIMPs, the energy scale for low energy nuclear recoils must be well characterized. The reasons for this can be found in the differential rate of WIMP-induced nuclear recoils as a function of recoil energy $Q$, which is expressed for spin-independent (SI) interactions in Eq. 2.1 as the product of four components [5]:

$$
\frac{d R}{d Q}=\frac{\rho_{0}}{m_{\chi}} \times \frac{\sigma_{0} A^{2}}{2 m_{p}^{2}} \times F^{2}(Q) \times \int_{v_{m}}^{v_{e s c}} \frac{f(v)}{v} d v
$$

The first term in Eq. 2.1 is the number density of WIMPs, with $\rho_{0}$ as the mass density of dark matter in the solar system and $m_{\chi}$ as the dark matter mass. The second term is the particle physics 
component, where $\sigma_{0}$ is the cross section for interactions on nucleons, $A$ is the atomic mass of the target, and $m_{p}$ is the mass of a proton. SI interactions benefit from the assumption that all nucleons contribute coherently to the rate; this coherence leads to an $A^{2}$ enhancement, written here explicitly, which is one reason xenon is such a good target for dark matter searches. However, the interaction loses its coherence as the recoil energy $Q$ gets large (effectively the de Broglie wavelength becomes small relative to the size of the nucleus). The third term in Eq. 2.1 is the nuclear form factor, $F^{2}(Q)$, accounting for this loss of coherence. To first order, the form factor can be described as a falling exponential, $F^{2}(Q)=\exp \left(-Q / Q_{0}\right)$, with $Q_{0}$ an energy scale that is smaller for larger nuclei (i.e. larger nuclei lose coherence at lower recoil energies). The final term is an integral over the velocity distribution of dark matter in the solar system, as faster WIMPs are more likely to deposit a meaningful amount of energy in an elastic scatter. The integral has a kinematic lower cutoff set by the minimum velocity for a WIMP with mass $m_{\chi}$ required to produce a nuclear recoil with energy $Q$,

$$
v_{\text {min }}=\sqrt{\frac{Q\left(A+m_{\chi}\right)^{2}}{2 m_{\chi}^{2} A}} .
$$

The higher cutoff, $v_{e s c}$, is the escape velocity; WIMPs moving faster than $v_{e s c}$ are not bound in the galaxy.

Because of the form factor and velocity distribution terms, the rate of WIMP-induced nuclear recoils falls exponentially with recoil energy. This exponential fall is particularly acute for low mass dark matter. Understanding the response of the detector at low energies is therefore critical to determining the dark matter sensitivity. Beyond the signal yield, the sensitivity of a detector to light dark matter is also subject to systematic errors because of the velocity distribution term. Most calculations of dark matter rates assume WIMP velocities follow a Maxwell-Boltzmann halo model [6], but several authors have suggested that this model is a simplified picture of the Milky Way halo [7]. Even within the standard halo model, calculated rates can be highly dependent on $v_{e s c}$, an astronomical observable with non-zero uncertainties. Figure 1 shows $f(v)$ for the standard Maxwell-Boltzmann halo model for two values of $v_{e s c}\left(v_{e s c}=544 \mathrm{~km} / \mathrm{s}\right.$ is currently the most widely used value). The red line shows $v_{\min }$ for a $9 \mathrm{GeV}$ WIMP interacting in a pure xenon detector with an energy threshold of $3 \mathrm{keV}$ (the assumed energy threshold in the LZ Conceptual Design Report (CDR) [8]). Ignoring effects of energy resolution, the xenon detector is only sensitive to the region to the right of the red line, representing $<1 \%$ of the entire distribution. By contrast, the magenta line shows $v_{\min }$ for a neon target, sensitive to $27 \%$ of the distribution. Changes in either the energy scale, WIMP mass, or the halo model, particularly in the tails of the distribution, can have large effects on the predicted dark matter rate. To give one example, if $m_{\chi}=7.9 \mathrm{GeV}$ or $Q=4 \mathrm{keV}, v_{\text {min }}$ for xenon is greater than $544 \mathrm{~km} / \mathrm{s}$.

\section{Adding He or $\mathrm{Ne}$}

The kinematics described in the previous section show that the two ways to search for light dark matter are with a light target or low energy thresholds, and doping LXe-TPCs with $0.1-0.5 \%$ levels of helium and/or neon can satisfy both conditions. Importantly, because helium and neon have no long-lived radioactive isotopes, they can be added to a xenon detector without affecting the background levels. 


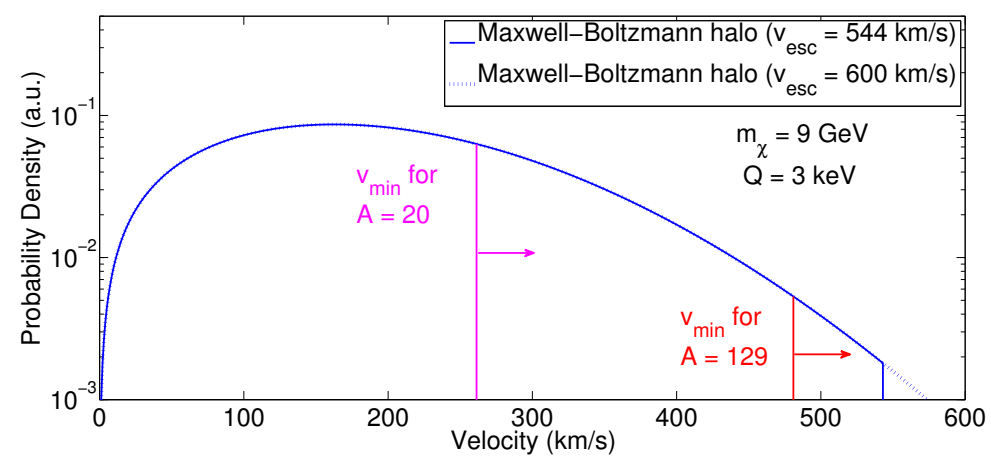

Figure 1: The velocity distribution, $f(v)$, for the standard Maxwell-Boltzmann halo model of [6] for two values of $v_{\text {esc }}$. The red line shows $v_{\min }$ for a $9 \mathrm{GeV}$ WIMP interacting in a xenon detector with an energy threshold of $3 \mathrm{keV}$ (the assumed energy threshold in the LZ CDR). In that case, and ignoring energy resolution, a xenon detector would only be sensitive to the region to the right of the red line, representing $<1 \%$ of the entire distribution. By contrast, the magenta line shows $v_{\min }$ for a neon target, sensitive to $27 \%$ of the distribution. Changes in either the energy scale, WIMP mass, or the halo model, particularly in the tails of the distribution, can have large effects on the predicted dark matter rate. As an example, if $m_{\chi}=7.9$ $\mathrm{GeV}$ or $Q=4 \mathrm{keV}, v_{\min }$ for xenon would be pushed above $544 \mathrm{~km} / \mathrm{s}$.

The sensitivity of a Ne/He doped xenon detector depends on how much of the lighter noble gas can be loaded into the detector. Henry's Law states that the amount of gas dissolved in a liquid is proportional to the partial pressure of the gas above the liquid, and the proportionality constant is known as the Henry coefficient. As the partial pressure of helium or neon gas that can be added will be limited by mechanical considerations as well as operational requirements, the Henry coefficient will be the main factor in determining the ultimate sensitivity. There are no published data on the Henry coefficients for helium or neon in LXe. Unpublished measurements within the LUX collaboration and confirmed in a simple test stand at Fermilab show that helium can be loaded into xenon at the level of $0.1-0.3 \%$ at 1 bar of partial pressure [9]. Measurements in argon show that neon dissolves about 5 times more readily than helium [10]. For now, we assume that levels of $0.1 \%$ and $0.5 \%$ doping are achievable for $\mathrm{He}$ and Ne respectively. These numbers need to be measured systematically in liquid xenon as a function of temperature before the idea presented here could be executed.

A well-known problem with PMTs is that helium can diffuse through PMT glass, leading to afterpulsing as the electron cascade ionizes He atoms that drift back to the cathode and initiate a second pulse. If the partial pressure of $\mathrm{He}$ in the tube is too high $\left(1 \times 10^{-3}\right.$ torr $)$, significant afterpulsing is observed, and the tubes become unusable at ten times that pressure [11]. The rate of diffusion is proportional to the partial pressure of $\mathrm{He}$ in the environment, but it is exponentially suppressed by temperature via the Arrhenius relationship. The Hamamatsu R11410 PMTs used in both XENON1T and LZ have a relatively thick face and a metal body, which also decreases the diffusion rate. Based on the diffusion coefficients measured by Altemose [12], it would take the these PMTs ten years in a 1 bar helium environment at $165 \mathrm{~K}$ to develop $1 \times 10^{-3}$ torr of partial pressure (with neon suppressed even further), but these numbers need to be confirmed experimentally.

The most important question regarding a He/Ne doped xenon detector is how the scintillation 
and ionization properties will change relative to the pure LXe case. First, from a practical standpoint, it is important that the S1 and S2 signals in doped LXe be at the same wavelength as in pure LXe and observable by the same PMTs. In noble liquid gases, scintillation is produced by the decay of metastable molecular states. The amount of energy required to form these molecules decreases for increasing $\mathrm{Z}$, and the wavelength of pure helium and neon scintillation is much deeper in the UV than xenon light [13]. Data from mixtures with $\sim 10 \mathrm{ppm}$ xenon in liquid argon show that excitations can be efficiently transferred from argon to xenon, with the resulting light emission emitted at xenon wavelengths [14]; energy transfers from excitations of the heavy noble elements to lighter ones do not take place because they are not energetically favorable. Therefore, the wavelength of S1/S2 light in a doped LXe-TPC will be unchanged. A second point is that because the liquid environment will still be dominated by Xe atoms (with their large numbers of bound electrons), the drifting of free electrons through the TPC will also be unaffected. In the gas phase, where the S2 amplification occurs, excitations of the lighter elements in the gas phase should efficiently transfer their energy to the heavier gas atoms, leading to minimal overall change in gain. A preliminary simulation of the mixture of gases in the Garfield simulation package [15] confirms this expectation.

Given the importance of both energy thresholds and ER/NR discrimination, there are two major unknowns regarding the scintillation and ionization properties of a $\mathrm{He} / \mathrm{Ne}-\mathrm{LXe}$ mixture. First, what are the expected S1 and S2 yields for He/ $\mathrm{Ne}$ recoils in LXe? Electrons deposit their energy primarily into electronic excitations (electronic stopping) while xenon recoils in LXe deposit their energy into both electronic excitations and elastic collisions with nuclei (nuclear stopping). All the electronic energy is eventually collected as signal, but some of the energy given to nuclear recoils is lost as heat. Calculating the final electronic energy deposition from a xenon recoil is more complicated than simply taking the amount given directly from the primary recoil to electronic excitations, as secondary nuclei from the nuclear collisions in turn partition their energy into electronic and nuclear stopping, but Lindhard theory [16] gives an approximation for the "Lindhard factor", or the total electronic energy deposition from nuclear recoils relative to electronic recoils of the same energy. Figure 2 shows a plot of the Lindhard factor vs. energy for xenon, and the signal produced by low energy xenon recoils is less than $20 \%$ that produced by ER of the same energy.

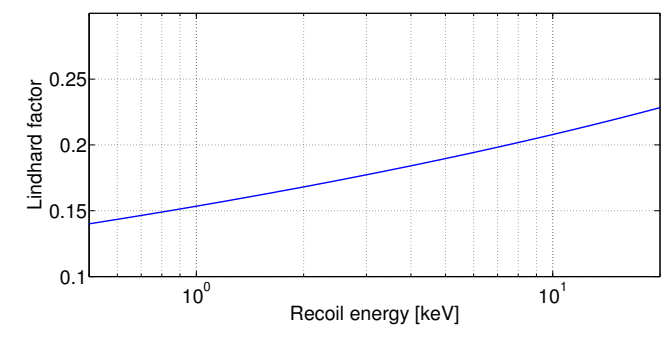

Figure 2: Fraction of energy going into observable signal (Lindhard factor) vs. recoil energy for xenon recoils in LXe.

\begin{tabular}{|c|c|c|}
\hline Recoil & Lindhard & SRIM \\
\hline Xenon & 0.02 & 0.02 \\
Neon & 0.20 & 0.09 \\
Helium & 0.68 & 0.69 \\
\hline
\end{tabular}

Table 1: Estimated fraction of energy given to electronic stopping for nuclear recoils (not accounting for secondary cascades) from $\mathrm{Xe}, \mathrm{He}$, and $\mathrm{Ne}$ recoils in LXe, calculated using Lindhard theory [17] or the SRIM simulation package [18].

Because helium and neon are so much lighter than xenon, they will not lose as much energy 
in elastic collisions with xenon atoms, leaving more energy for electronic excitation and a correspondingly larger signal. Simple approximations for the Lindhard factor do not exist for nuclei moving through fluids composed of a different element, but one can estimate the raw stopping powers (before accounting for the secondary cascades) using either Lindhard theory [17] or the SRIM simulation package [18]. Table 1 shows the predicted amount of energy going directly from the primary recoil into electronic stopping from $5 \mathrm{keV} \mathrm{Xe,} \mathrm{He,} \mathrm{and} \mathrm{Ne}$ recoils in LXe calculated via both methods. Neon and especially helium have a much larger fraction of energy deposited directly to electrons, i.e. directly into signal, without accounting for the secondary cascades that can only increase these fractions. It should be noted that the effect of the cascades will be reduced for neon and helium because they will not efficiently transfer energy to the predominantly xenon atoms around them, leading to more sub-ionization energy depositions. Even so, one can expect larger signals (both charge and light) from helium and neon recoils in LXe than from xenon recoils, and a correspondingly lower energy threshold.

The second key question is how will that increased signal be partitioned into S1 and S2; what happens to the S2/S1 ratio that is so important for rejecting electron recoil backgrounds in LZ? Given that the ratio is determined by track structures, and recoiling electrons will still be interacting with xenon atoms, the S2/S1 ratio for electrons should be unchanged. As it is not fully understood what drives the partitioning between $\mathrm{S} 1$ and $\mathrm{S} 2$ for xenon recoils in LXe, the most that can be said here is that $\mathrm{He} / \mathrm{Ne}$ recoils will likely lie below the electron band. As one example, in scintillating $\mathrm{CaWO}_{4}$ crystals operated by the CRESST dark matter experiment, oxygen recoils produce a light/heat ratio that lies between the electron and tungsten recoil bands [19]. One can imagine being able to separate the $\mathrm{He} / \mathrm{Ne}$ recoil bands from the xenon and ER bands in the doped LXe, but the ultimate level of discrimination power in a He/Ne experiment is unknown.

Doping LZ (for the purposes of argument) with $\mathrm{He} / \mathrm{Ne}$ has other attractive features. By using $\mathrm{LZ}$ as the detector, the $\mathrm{He} / \mathrm{Ne}$ target benefits from the self-shielding provided by a large LXe volume. A $1 \mathrm{MeV}$ gamma ray has an attenuation length of about $6 \mathrm{~cm}$ in LXe, which is less than $1 / 10$ the design radius of LZ. The ratio of attenuation length to radius for the same gamma ray in a $50 \mathrm{~kg}$ neon or helium detector would be 0.6 and 2.6, respectively, and self-shielding would not be effective. Second, for very low energy depositions like the ones relevant for light WIMP searches, events near detector surfaces can produce significant and difficult to understand backgrounds. For example, the CoGeNT detector observed an increasing spectrum at threshold that has been interpreted as both a dark matter signal but also as a poorly modeled surface background [20,21]. For a He/Ne run in LZ, these surface effects disappear with XYZ-reconstruction, allowing for much cleaner searches at low energies.

Another possibility in a doped LXe-TPC is the potential for doing "S2-only" analyses down to very low thresholds, as pioneered by the XENON10 experiment [22]. Because LXe-TPCs are sensitive to single electrons, and the ionization energy is less than $100 \mathrm{eV}, \mathrm{S} 2$-only analyses can probe energy thresholds as low as a few hundred $\mathrm{eV}$. Given the increased charge yield expected from $\mathrm{He}$ and Ne recoils in LXe, S2-only searches should be even more effective in a doped detector. By combining a He target with a few hundred $\mathrm{eV}$ threshold and the self-shielding of LXe, LZ will be sensitive to WIMP masses well below $1 \mathrm{GeV}$.

Like other ultra-low-threshold experiments such as CoGeNT, DAMIC, and CDMS-lite, S2only searches give up discrimination to achieve such low thresholds. Despite the lack of back- 
ground rejection, these searches can be competitive because the low WIMP mass region of parameter space is still relatively unexplored and requires less stringent background levels than the high mass region. By the same token, low mass searches do not require several tonnes of target mass to make progress, and $\sim 10 \mathrm{~kg}$ of target can provide orders of magnitude increase in sensitivity over current results. Doping at the sub-percent level in LZ will achieve He/Ne target masses in this range.

Figure 3 shows a preliminary estimate of the sensitivity of a 2 year run with $5(25) \mathrm{kg}$ of $\mathrm{He}(\mathrm{Ne})$ loaded into LZ assuming the same background levels as in the LZ CDR and a conservative analysis that does not incorporate any background subtraction. The sensitivity scales linearly with the mass of helium or neon that can be dissolved in liquid xenon. The projections assume that for neon and helium respectively, $10 \%$ and $0 \%$ of the energy lost to nuclear stopping ultimately becomes electronic excitations, leading to factors of 1.5 and 3 increase in signal relative to xenon recoils, partitioned equally between $\mathrm{S} 1$ and $\mathrm{S} 2$. The projections assume 99.5\% discrimination against electron recoils. For the helium S2-only analysis, because of the very low energies involved, the dominant background is ${ }^{8} \mathrm{~B}$ solar neutrinos coherently scattering with xenon atoms, and the assumed exposure is only $1 / 100$ that of the other projections before the search becomes background limited (assuming no background subtraction). Because the ${ }^{8} \mathrm{~B}$-Xe spectrum falls sharply with energy, the projected sensitivity of the He S2-only search depends strongly on the assumed charge yield for He recoils.

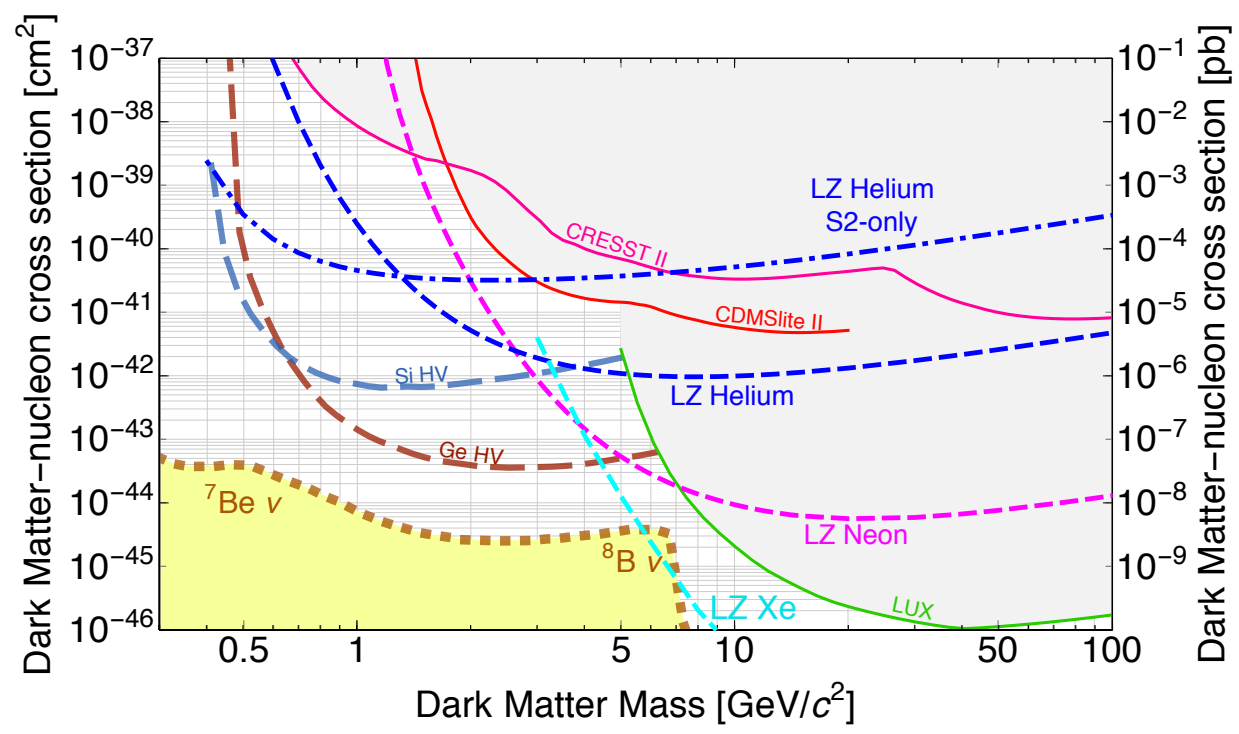

Figure 3: The dashed blue and magenta curves show projected sensitivity of a 3 year run with $5(25) \mathrm{kg}$ of $\mathrm{He}(\mathrm{Ne})$ loaded into LZ assuming the same backgrounds as in [23], with xenon-only sensitivity shown in light blue. The projections are based on conservative Poisson assumptions about the backgrounds and do not take advantage of profile or maximum likelihood analysis methods. The increased reach provided by helium and neon make a good complement to the projected SuperCDMS-SNOLAB sensitivity, Ge HV and Si HV projections shown in dashed brown and blue respectively [24]. The current best low mass limits, from CRESST II and CDMS-lite, are also shown [25, 26]. 


\section{Proposed measurements at a neutron beam}

As the ultimate science reach depends on the amount of scintillation and ionization produced in doped LXe, a detailed study of these properties is required before this idea could be carried out. These properties could measured in an experiment similar to the Scintillation Efficiency of Noble Elements (SCENE) experiment, in which the nuclear recoil response in a liquid argon TPC was measured at the neutron beam at the Nuclear Science Laboratory of the University of Notre Dame [27, 28]. The neutron beam at Notre Dame is a pulsed, tunable, mono-energetic neutron beam that is ideal for measuring low energy nuclear recoils in liquid noble gases. Using secondary detectors located at fixed angles to the beam axis as a tag, one can pick out xenon recoils of a known energy in the liquid. After making an initial suite of measurements in pure xenon, helium and neon could be added to the detector in turn to measure the response to the lighter nuclei. Experimentally, $\mathrm{He}$ and Ne recoils will be easily identified, as for the same neutron scattering angle, the energy deposited to $\mathrm{He}(\mathrm{Ne})$ will be increased relative to xenon by a factor of 20(6). The scattering cross sections for $\mathrm{Xe}, \mathrm{Ne}$, and $\mathrm{He}$ for $1 \mathrm{MeV}$ neutrons are within factors of two [29, 30]. Figure 4 shows results from a Geant4 simulation [32] of the recoil spectra in a He-doped LXe detector for two beam/angle configurations including backgrounds, showing how the helium energy peak pokes out at higher recoil energies above the main xenon "wall" at lower energies. When the expected larger signal yield is included, it should be relatively straightforward to identify the component created by the lighter element. Preliminary calculations based on the experience with SCENE and a similar measurement with $\mathrm{NaI}$ [31] suggest that even with the low doping fraction of $\mathrm{He}$ in $\mathrm{LXe}$, adequate statistics on He recoils can be collected in a week-long run.

\section{Conclusion}

The nature of dark matter remains a mystery despite the progress made in the past two decades. The LZ detector and other LXe-TPCs will improve sensitivity by two orders of magnitude but as currently designed are limited to being single-target experiments. The addition of small quantities of helium or neon can extend the flexibility of these detectors, increasing the sensitivity to lower dark matter masses, by making them less susceptible to systematic uncertainties associated with WIMP kinematics or recoil energy scales and searching for dark matter with multiple targets in the same apparatus. This approach makes use of the excellent self-shielding properties of LXe, while incorporating light targets that are ideal for a light WIMP search. The projected reach of this idea is complementary to that of the SuperCDMS-SNOLAB experiment and would be critical in the event of any discovery. A helium or neon run of LZ would deliver excellent sensitivity at a fraction of the cost of a dedicated detector. 

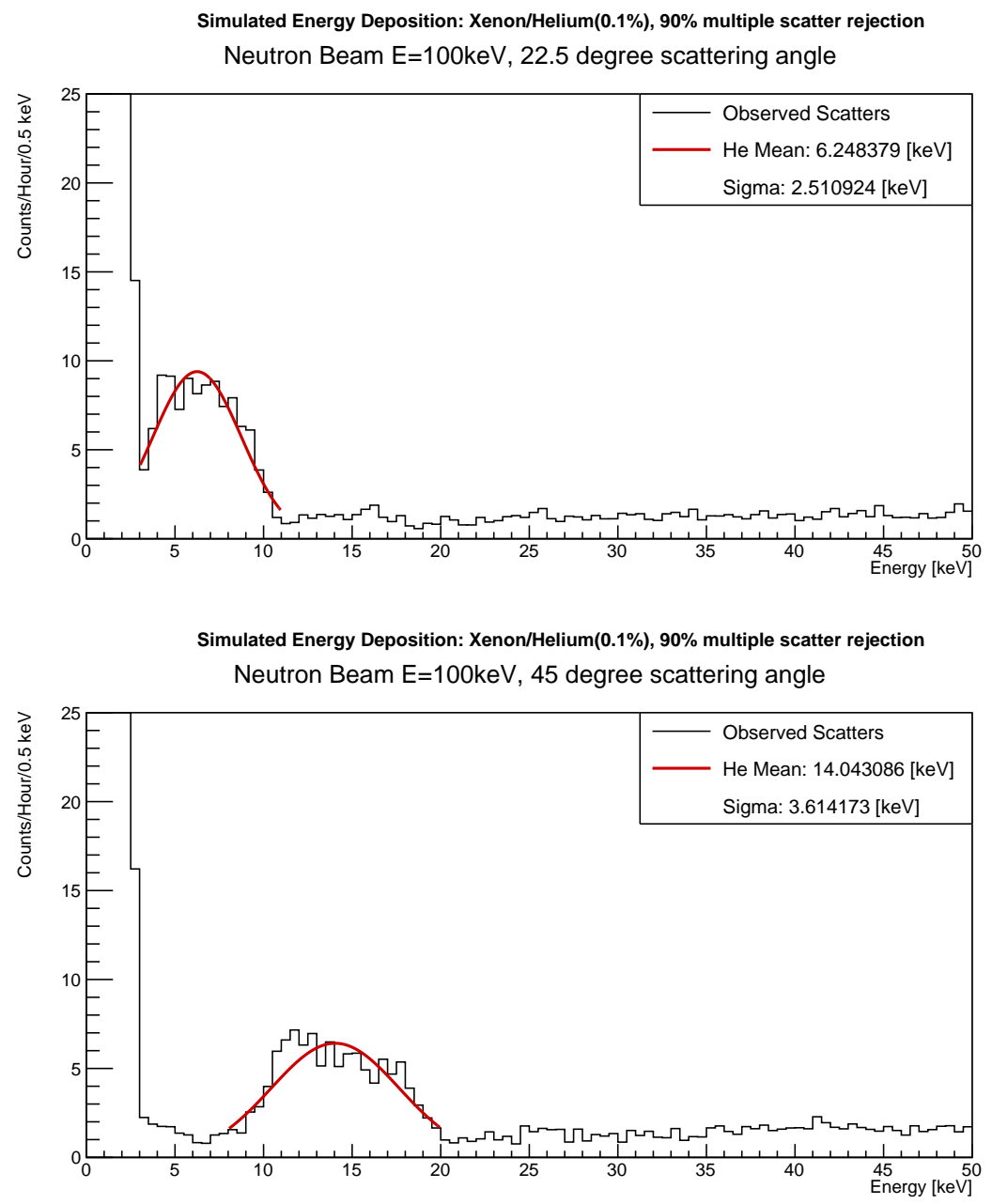

Figure 4: Results of Geant4 simulations of two different configurations of the neutron beam and tagging detector. In both cases $(6.25 \mathrm{keV}$ and $14 \mathrm{keV}$ helium recoils), the large increase at very low energies is the dominant xenon recoil signal. However, the helium peak is clearly observable for both cases. The simulated live time in these simulations is approximately one day of beam at Notre Dame. 


\section{References}

[1] J. Kozaczuk and S. Profumo, "Light NMSSM neutralino dark matter in the wake of CDMS II and a 126 GeV Higgs boson," Phys. Rev. D 89, 095012 (2014); D. G. Cerdeño, J.-H. Huh, M. Peiró, and O. Seto, "Very light right-handed sneutrino dark matter in the NMSSM," J. Cosmol. Astropart. Phys. 2011, 027 (2011).

[2] T. Lin, H.-B. Yu, and K. M. Zurek, "Symmetric and asymmetric light dark matter," Phys. Rev. D 85, 063503 (2012).

[3] J. Fan, M. Reece, L.-T. Wang, "Non-relativistic effective theory of dark matter direct detection," J. Cosmol. Astropart. Phys. 2011, 042 (2010); A. L. Fitzpatrick, W. C. Haxton, E. Katz, N. Lubbers, and Y. Xu, " The effective field theory of dark matter direct detection," J. Cosmol. Astropart. Phys. 2013, 004 (2013); N. Anand, A. L. Fitzpatrick, W. C. Haxton, "Weakly interacting massive particle-nucleus elastic scattering response," Phys. Rev. C 89, 065501 (2014); M. I. Gresham and K. M. Zurek, "Effect of nuclear response functions in dark matter direct detection," Phys. Rev. D 89, 123521 (2014).

[4] V. Gluscevic, M. I. Gresham, S. D. McDermott, A. H. G. Peter, and K. M. Zurek, "Identifying the Theory of Dark Matter with Direct Detection," J. Cosmol. Astropart. Physics 2015, 057 (2015).

[5] G. Jungman, M. Kamionkowski, and K. Griest, "Supersymmetric Dark Matter," Phys. Rep. 267, 195 (1996); G. Bertone, D. Hooper, and J. Silk, "Particle dark matter: evidence, candidates and constraints," Phys. Rep. 405, 279, (2005); J.L. Feng, "Dark Matter Candidates from Particle Physics and Methods of Detection," Ann. Rev. Astr. Astroph., 48, 496 (2010).

[6] J. D. Lewin and P. F. Smith, "Review of mathematics, numerical factors, and corrections for dark matter experiments based on elastic nuclear recoil," Astrop. Phys. 6, 87 (1996).

[7] M. Kuhlen, N. Weiner, J. Diemand, P. Madau, B. Moore, et. al., "Dark matter direct detection with non-Maxwellian velocity structure," J. Cosmol. Astropart. Phys. 2010, 030 (2010); M. Lisanti, L. E. Strigari, J. G. Wacker, and R. H. Wechsler, "Dark matter at the end of the Galaxy," Phys. Rev. D 83023519 (2011); Y.-Y. Mao, L. E. Strigari, R. H. Wechsler, H.Y. Wu, and O. Hahn, "Halo-to-halo similarity and scatter in the velocity distribution of dark matter," Astrophys. J. 764, 35 (2013); J. Read, G. Lake, O. Agertz, and V. P. Debattista, "Thin, thick and dark discs in $\Lambda$ CDM," Mon. Not. Roy. Astron. Soc. 389, 1041 (2008); J. Read, L. Mayer, A. Brooks, F. Governato, and G. Lake, "A dark matter disc in three cosmological simulations of Milky Way mass galaxies," Mon. Not. Roy. Astron. Soc. 397, 44 (2009); C. W. Purcell, J. S. Bullock, and M. Kaplinghat, "The dark disk of the milky way," Astrophys. J. 703, 2275 (2009); F. Ling, E. Nezri, E. Athanassoula, and R. Teyssier, "Dark matter direct detection signals inferred from a cosmological N-body simulation with baryons," J. Cosmol. Astropart. Phys. 2010012 (2010);

[8] LZ Collaboration: Akerib et al., "LUX-ZEPLIN (LZ) Conceptual Design Report," arXiv:1509.02910 (2015). 
[9] A. Dobi, LUX Collaboration, Private communication.

[10] F. E. Karasz and G. D. Halsey Jr., "Solubility of Helium and Neon in Liquid Argon. An Approximation to the Entropy of Lattice Vacancy Formation in Liquid Argon," J. Chem. Phys. 29, 173 (1958).

[11] C. C. Garrard, R. M. McAlpine, and A. G. Wright, Electron Tubes Technical Reprint R/P101, (2012).

[12] V. O. Altemose, "Helium Diffusion through Glass,"J. App. Phys. 32, 1309 (1961).

[13] E. Aprile, A.E. Bolotnikov, A.I. Bolozdynya and T. Doke, Noble Gas Detectors, Wiley-VCH Verlag, (2006).

[14] C. G. Wahl, E. P. Bernard, W. H. Lippincott, J. A. Nikkel, Y. Shin, D. N. McKinsey, "Pulseshape discrimination and energy resolution of a liquid-argon scintillator with xenon doping," J. Inst. 9, P06013 (2014); P. Peiffer et al., "Pulse shape analysis of scintillation signals from pure and xenon-doped liquid argon for radioactive background identification," J. Inst. 3, P08007 (2008); S. Kubota, M. Hishida, M. Suzuki, and J. Ruan, " Liquid and solid argon, krypton and xenon scintillators," Nuc. Instrum. Meth. 196, 101 (1982).

[15] R. Veenhof, "Garfield - simulation of gaseous detectors, http://cern.ch/garfield".

[16] J. Lindhard, M. Scharff, and H. E. Schiott., "Range concepts and heavy ion ranges (Notes on atomic collisions II), Mat. Fys. Medd. Dan. Vid. Selsk., bf 33, no. 10 (1963).

[17] J. Lindhard and M. Scharff, "Energy Dissipation by Ions in the keV Region," Phys. Rev. 124, 128 (1961).

[18] J.F. Zeigler et al., Stopping Range of Ions in Matter, www.srim.org.

[19] G. Angloher et al., Eur. Phys. J. 72, 1971 (2012).

[20] C. E. Aalseth et al., "CoGeNT: A search for low-mass dark matter using p-type point contact germanium detectors," Phys. Rev. D88, 012002 (2013).

[21] C. Aalseth et al., "Maximum Likelihood Signal Extraction Method Applied to 3.4 years of CoGeNT Data," arXiv:1401.6234 (2014).

[22] J. Angle et al., "Search for Light Dark Matter in XENON10 Data," Phys. Rev. Lett. 107, 051301 (2011).

[23] J. Dobson for LZ Collaboration, "Overview and status of LUX-Zeplin", Identification of Dark Matter 2016 (2016).

[24] SuperCDMS Collaboration: Agnese et al., "Projected Sensitivity of the SuperCDMS SNOLAB experiment," arXiv:1610.00006 (2016).

[25] CRESST Collaboration: Angloher et al., "Results on light dark matter particles with a lowthreshold CRESST-II detector," Eur. Phys. J C 76, 25 (2016). 
[26] SuperCDMS Collaboration: Agnese et al., "WIMP-Search Results from the Second CDMSlite Run," Phys. Rev. Lett. 116, 071301 (2016).

[27] T. A. Alexander et al., "Observation of the Dependence of Scintillation from Nuclear Recoils in Liquid Argon on Drift Field," Phys. Rev. D 88, 092006 (2013).

[28] T. A. Alexander et al., "Measurement of scintillation and ionization yield and scintillation pulse shape from nuclear recoils in liquid argon," Phys. Rev. D 91, 092007 (2015).

[29] M. B. Chadwick et al., "ENDF/B-VII.1 Nuclear Data for Science and Technology: Cross Sections, Covariances, Fission Product Yields and Decay Data," Nuclear Data Sheets 112, 2887 (2011): http://www.nndc.bnl.gov/sigma/index.jsp.

[30] S. MacMullin, M. Kidd, R. Henning, W. Tornow, C.R. Howell, and M. Brown, "Measurement of the elastic scattering cross section of neutrons from argon and neon," Phys. Rev. C 87, 054613 (2013).

[31] J. Xu et al., "Scintillation efficiency measurement of $\mathrm{Na}$ recoils in $\mathrm{NaI}(\mathrm{Tl})$ below the DAMA/LIBRA energy threshold," Phys. Rev. D 92, 015807 (2015).

[32] S. Agostinelli et al, "Geant4: a simulation toolkit," Nucl. Instr. Meth. A 506 250-303 (2003); 